\title{
Motion Planning of Vehicles for Crash Mitigation in Emergency Driving Scenarios
}

\author{
Daofei $\mathrm{Li}^{\star}$ and Zhaohan $\mathrm{Hu}$
}

\begin{abstract}
Motion planning in dynamic environment is crucial to the automated driving safety. In extremely emergency scenarios with unavoidable collisions, especially those with complex impact patterns, the potential crash risk should be well considered in motion planning. This paper proposes a motion planning algorithm for unavoidable collisions, which directly embeds a generalized crash severity index model to vehicle-to-vehicle collisions of multiple impact patterns. Firstly, the clothoid curve is used to sample the vehicle trajectory before collision, and a two-degree-of-freedom model is adopted to predict the vehicle poses corresponding to each sample path. Then, the crash severity index model is to estimate the potential crash severity of all sample paths. To improve the inferring time efficiency, a neural network is constructed and deployed to approximate the nonlinear severity model. Finally, the crash-severity-optimal trajectory is tracked through model predictive control method. Results show that by combining the braking and steering interventions for better crash severity reduction, the proposed strategy can achieve better mitigation effects than commonly-used collision-avoidance strategies. The deployment of real car experiment and sensitivity analysis demonstrate that the planning algorithm can guarantee real-time and reliably safe performances.
\end{abstract}

Index Terms-Automated driving, Collision avoidance, Crash mitigation, Crash severity, Motion planning

\section{INTRODUCTION}

$\mathrm{M}$ OTION planning for automated driving has come a long way. Due to the fast-changing constraints and the varying uncertainties, motion planning in dynamic scenes has become far more challenging than that in near-static scenes. A common solution is to improve the computational frequency of a planning algorithm, so as to divide the dynamic problem into a finite number of static sub-problems. Thanks to the cost-effective computing capability available recently, motion planning algorithms to attack some dynamic scenes are becoming ready for the Advanced Driving Assist System

This work has been submitted to the IEEE for possible publication. Copyright may be transferred without notice, after which this version may no longer be accessible.

Manuscript submitted November 20th, 2021. This work is supported by Department of Science and Technology of Zhejiang (Proposal No. 2021C01SA601840). (Corresponding author: Daofei Li.)

The authors are with Institute of Power Machinery and Vehicular Engineering, Zhejiang University, Hangzhou, 310027, China (e-mail: dfli@zju.edu.cn; zhhu@zju.edu.cn).
(ADAS) market, namely Levels 1-2 according to SAE J3016, and even for Level 3 driving automation, too.

Highly dynamic driving situations occur frequently in daily driving, especially in urban and rural areas. The emergency scenarios may be due to reckless driving, driver visual occlusion, etc. Fig. 1 presents one example of ramp-merging interaction driving, in which the merging vehicle is too aggressive and the ego vehicle may need to yield by emergency braking or steering. In extreme situations without enough space or time to stop, even if the ego vehicle is fully-autonomous, no matter what evasive measures are taken, the collision accident cannot be fully avoided. As in Automated Emergency Braking (AEB) and Automated Emergency Steering (AES), the complete avoidance of a potential collision is only possible in slightly dynamic scenes, with a typical maximum velocity of activation as $50 \mathrm{~km} / \mathrm{h}$. In cases with braking-only ADAS, although collision avoidance fails, by reducing the vehicle velocity the late-coming activation of AEB may still contribute to mitigation of crash injury. However, when it comes to evasive steering, since the vehicle direction and speed are both controlled within very strict constraints of time and space, the activation of AES functions is asking for a comprehensive and reliable algorithm of motion planning.

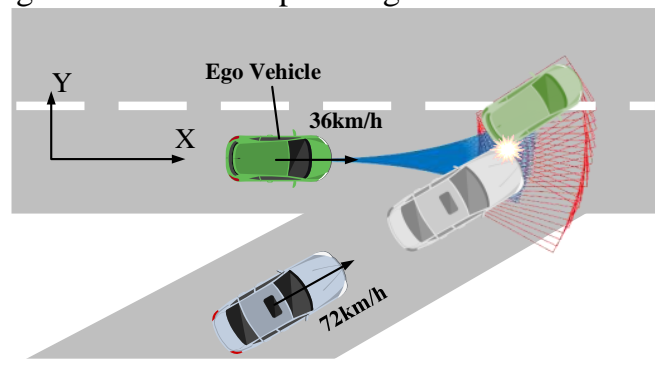

Fig. 1. An Emergency Scenario in Entry Ramp Merging

On the other hand, most motion planning algorithms focus on how to avoid obstacles in potentially dangerous scenarios [1-6], while very few have contributed to how to reduce the crash injury in unavoidable collisions via motion planning [7-11]. For the example in Fig. 1, AEB-only strategy is to stop the ego vehicle as soon as possible, but for AES-only strategy, an optimal path to avoid a collision may not exist and a sub-optimal path with the least crash injury needs to be found from the sample paths in light blue. According to vehicle collision dynamics theories [12], the states of vehicles at the collision-imminent moment, including the longitudinal velocities and the relative pose of both vehicles, have great effects on the final collision injury results. Sometimes a failed attempt to avoid a collision, by AEB, AES or both, does not 
lead to the least crash injury. Therefore, the motion planning problem in unavoidable collision scenarios cannot be solved simply by the existing collision-avoidance-oriented approaches. To achieve zero road fatality, namely Vision Zero, the research community should try best in mitigating risk during all phases of collision, even in the last half second before collision. As for safer motion planning, there are further needs to tackle the challenge of risk mitigation in unavoidable collisions.

The main contributions of this paper are as follow.

(1) We propose a motion planning scheme to handle the crash mitigation in unavoidable collisions, which includes a generalized crash severity index model suitable for various collision patterns. To accelerate the inferring time efficiency, a neural network is constructed and deployed to solve the nonlinear crash severity model equations.

(2) Simulations show that by considering crash severity our planning algorithm can achieve better crash mitigation performances than current collision-avoidance-oriented algorithms. This further reveals that a new mindset of comprehensive safety strategy should not focus only on collision avoidance, but also the last resort of crash mitigation if collision is unavoidable.

(3) Real vehicle experiment and sensitivity analysis are carried out, which demonstrate that the proposed algorithm can be deployed real-time and provide reliably safe performances under conditions with model uncertainties.

The rest of the paper is organized as follows. Section II summarizes related work on collision avoidance planning methods, crash severity models and their applications in motion planning. Section III presents the entire crash mitigation algorithm structure in which the proposed planning approach fits in. Section IV details the planning algorithm, including path sampling, the generalized crash severity index and its real-time solution. The proposed solution is validated through simulation and vehicle experiment in Section V. The conclusions and possible improvements to applications are presented in Section VI.

\section{RELATED WORK}

Research on motion planning for collision avoidance has achieved several types of solutions, namely graph search based, sampling based, interpolating curve based and numerical optimization based approaches [1]. For example, the graph search based algorithms like $\mathrm{A}^{*}$ [13] and sampling based algorithms like RRT [14] can be used to select key points along the desired path, then the local path planning is conducted by fitting the curves with polynomials [15] or other interpolating curves. Different curves are used to better fit the kinematics characteristics of different vehicles. Some researchers use the Dubins curve, an asymmetric curve, to smooth the piecewise path generated by algorithms like RRT [16]. For simple and effective solutions, special curves are used to fit the generated path, e.g. the Reeds-Shepp curve that is widely used in parking scenarios [17]. As a popular numerical optimization based approach, artificial potential field is also extensively adopted to model the potential energy of different obstacles and then to find a target path with the lowest collision risk along the path
[6]. In addition to [1], thorough literature reviews in this field can be further referred to the recently-published [2] and [3].

Among the existing literature, there are many collisionavoidance-oriented motion planning algorithms that consider the crash severity. For instance, a study uses predictive occupancy map to segment the road into different possible collision severity level parts, and then motion planning is conducted based on the map that indicates the severity level of different areas [4]. To extend the commonly-used forward collision avoidance capability, [5] proposes a 360-degree planning algorithm to avoid potential crashes from all horizontal directions, which also uses predictive occupancy map for risk assessment in planning.

On the other hand, there are only a few reports that directly tackle the planning problem for unavoidable collision scenarios. It is partially due to the complexities of collision dynamics models, while for motion planning applications such models should be both accurate and computing-efficient. In fact, the crash severity level is an important topic in vehicle passive safety research, including those theoretical and engineering crashworthiness models [12]. Theoretically, the vehicle collision dynamics can only be accurately described using complicated models, e.g. by finite element methods (FEM). On contrast, for rough crash injury level evaluations, two typical methods are the Delta- $\mathrm{V}$ method (i.e. the velocity change in a collision) and the equivalent energy speed (EES) method that estimates the energy change of vehicles [18]. Some further improvements are made through momentum change approach, i.e. the change of momentum is used as a key indicator of the total severity level [19]. In a study that uses the kinetic energy loss to estimate the crash severity, two weighting factors are used to respectively estimate the severity index of crossing scenarios and rear-end conflicts scenarios [20]. In [21], a dimensionless crash severity index is proposed to model the oblique collisions with a non-zero coefficient of restitution, which can accommodate more combinations of vehicle attributes and impact conditions, and the model is validated by comparing to the data from EuroNCAP. The authors further extended and applied this model in [22-23].

The Delta-V method is the most used crash severity models in vehicle motion planning. [7] solves the motion planning problem with an MPC framework, in which the cost function includes a potential crash severity index (PCSI) using weighted risks due to Delta- $\mathrm{V}$, relative heading angle and mass ratio. Using the PCSI based on only the relative heading angle, [8] adopts the Bezier curve to sample the target path in planning. Another recent study integrates collision avoidance and crash mitigation into a whole using a multi-objective switching mechanism, while for crash severity evaluation the collision angle is further considered for improving the Delta- $\mathrm{V}$ method [9].

Another approach to address the collision model accuracy issue is to exploit the learning approach based on the crash data from real world accidents or high-fidelity crash test simulations. [10] handles the collision mitigation planning using Crash Severity Maps from multiple offline FEM simulations, which contain different combinations of lateral offset and relative heading angles between vehicles. To minimize collision severity in planning, a data-driven model is presented via a trajectory library relating injury severity with impact location, 
which comes from real accident data [11]. The effectiveness of such learning-based models depends on how well the crash data can cover the real application scenarios, especially when the data come from real accident surveys.

For crash mitigation software development in automated driving, given acceptable accuracy and computing efficiency performances, a mechanism-based model of crash severity is more appealing than learning-based models. This can contribute to better efficiency of functional safety validation, especially during the simultaneous development processes of software and hardware for a new vehicle. Therefore, the generalized CSI model in [21] is introduced in this study.

\section{AlgORITHM StRUCTURE}

The objective is to develop an algorithm to handle the driving scenarios in which a collision cannot be avoided completely. As known to vehicle safety community, the active safety (e.g. ADAS) and passive safety (e.g. seat belt) technologies try to tackle the vehicle safety problem by preventing and mitigating a collision, respectively.

The idea in this paper falls somewhere in between these two methodologies, which integrates the collision mitigation measures in passive safety to active safety. With this, during the short time before a collision, usually less than 2.0 seconds, the vehicle can actively adapt its positions and poses to minimize the potential crash severity level after the collision. The overall algorithm structure is schemed in Fig. 2.

(1) The Perception and Motion Prediction modules are responsible for dynamic environment modelling, including the obstacle speed and trajectory prediction.

(2) The Trajectory Generation module outputs all reachable paths via curve sampling, and their corresponding longitudinal acceleration $a_{x}$ and steering angle $\delta$.

(3) The Ego State Prediction module outputs the possible vehicle positions and poses, based on a vehicle dynamics model. Then the Crash Detection module checks whether both vehicles will collide in the corresponding paths of prediction. Time to collision (TTC) is calculated assuming the two vehicles keep moving at current speeds and directions. The collision is treated as unavoidable if TTC is less than a set threshold $T^{*}$, and the Crash Mitigation is activated, otherwise the Planning for Collision Avoidance is activated.

(4) The Crash Severity Index Estimation module calculates the corresponding crash severity level for each sampled path and a path with the least crash-severity is selected in the Risk Evaluation and Trajectory Selection module.

(5) The Trajectory Tracking module is to output the desired vehicle steering angle and longitudinal acceleration according to an optimal trajectory from planning.

Since the vehicle-to-vehicle interaction process in such emergency scenarios is extremely short, it is challenging for all modules. During this short process, the moving obstacle is assumed to move with a known velocity, but its sensitivity analysis is also carried out. The main focus of this research includes the shaded modules, while the modules of Perception, Motion Prediction and Collision Avoidance are not covered with details.

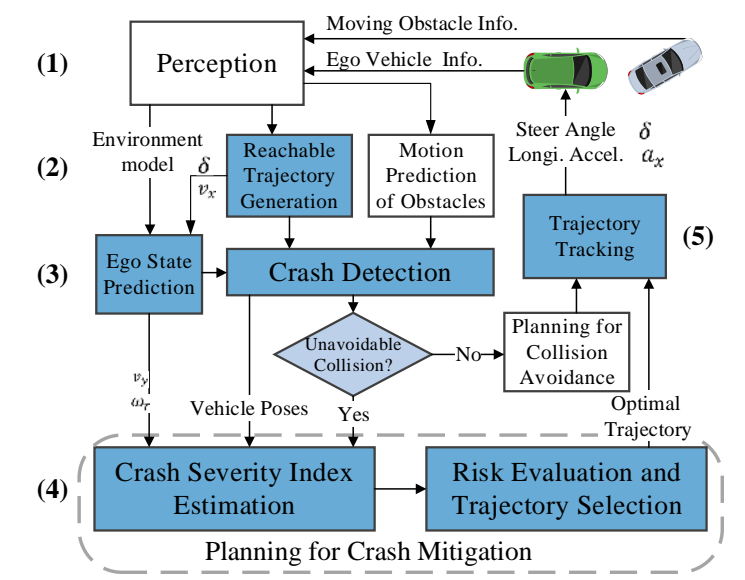

Fig. 2. Overall Structure of Crash Mitigation in Unavoidable Collisions

\section{Motion Planning And Control}

The proposed algorithm divides the crash mitigation problem into two steps: planning and control. Here we detail trajectory sampling, ego state prediction, crash severity estimation and trajectory tracking.

\section{A. Trajectory Sampling Using Clothoid Curves}

The clothoid curve, or Euler curve, has a wide range of applications in robotics since its curvature changes linearly along the length. The curvature at one specific point of a clothoid curve can be expressed as $K(s)=\kappa^{\prime} s+\kappa$, where $s$ represents the arc length, $\kappa$ and $\kappa^{\prime}$ are curve parameters. For a unit clothoid curve, the $x$ and $y$ coordinates in a planar coordinate system can be solved by integrating the current curvature and curvature changes as follow.

$$
\begin{aligned}
& x(s)=x_{0}+\int_{0}^{s} \cos \left(\kappa^{\prime} \tau^{2} / 2+\kappa \tau+\phi_{0}\right) \mathrm{d} \tau \\
& y(s)=y_{0}+\int_{0}^{s} \sin \left(\kappa^{\prime} \tau^{2} / 2+\kappa \tau+\phi_{0}\right) \mathrm{d} \tau
\end{aligned}
$$

where $\kappa^{\prime} s^{2} / 2+\kappa s+\phi_{0}$ is the angle at the point $s, \phi_{0}$ represents the initial angle. For the trajectory sampling problem here, every sampling starts from the initial coordinates of the vehicle, thus $\phi_{0}=0, x_{0}=y_{0}=0$.

Considering vehicle steering kinematics, the initial curvature $\kappa_{0}$ can be calculated from the current front wheel steering angle $\delta_{0}$, i.e. $\kappa_{0}=\tan \delta_{0} / \mathrm{L}$, where $\mathrm{L}$ is the vehicle wheelbase. From the starting position $(0,0)$, we generate totally 41 path samples for a given longitudinal acceleration profile. The corresponding initial front wheel steering angles are -20 to 20 degrees, with an increment of $1^{\circ}$ front wheel steering. For our vehicle example, the generated trajectory samples are shown in Fig. 3. For potential applications, the number of samples can be customized to accommodate steering limits and operational efficiency. 


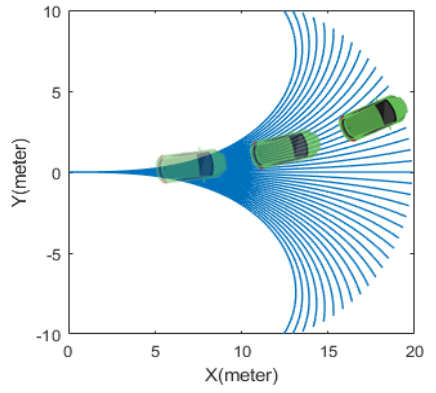

Fig. 3. Vehicle Trajectory Sampling Using Clothoid Curves

\section{B. Ego State Prediction by Vehicle Dynamic Model}

For each generated trajectory, it is necessary to predict the future positions and poses of the ego vehicle, for further crash severity analysis. Given vehicle initial states, the corresponding front wheel steering angle $\delta$ and speed $v_{x}$, the 2-DOF (Degrees of Freedom) vehicle dynamics model, is adopted to predict yaw rate $\omega_{r}$ and lateral velocity $v_{y}$ along the path.

The lateral and yaw dynamics are modelled as follows,

$$
\left\{\begin{array}{c}
\left(\mathrm{k}_{1}+\mathrm{k}_{2}\right) \frac{v_{y}}{v_{x}}+\left(\mathrm{ak}_{1}-\mathrm{bk}_{2}\right) \frac{\omega_{r}}{v_{x}}-\mathrm{k}_{1} \delta=\mathrm{m}\left(v_{y}+v_{x} \omega_{r}\right) \\
\left(\mathrm{ak}_{1}-\mathrm{bk}_{2}\right) \frac{v_{y}}{v_{x}}+\left(\mathrm{a}^{2} \mathrm{k}_{1}+\mathrm{b}^{2} \mathrm{k}_{2}\right) \frac{\omega_{r}}{v_{x}}-\mathrm{ak}_{1} \delta=\mathrm{I}_{\mathrm{z}} \dot{\omega}_{r}
\end{array},\right.
$$

where $\mathrm{k}_{1}$ and $\mathrm{k}_{2}$ represent the front and rear tire cornering stiffnesses, respectively. As shown in Fig. 4, C.G. indicates the center of gravity of vehicle, $\mathrm{m}$ is vehicle mass, $\mathrm{I}_{\mathrm{z}}$ is the rotational inertia of the vehicle, $a$ and $b$ represent the distances from C.G. to front and rear axles, respectively. $\omega_{r}$ is yaw rate, $v_{x}$ and $v_{y}$ are longitudinal and lateral velocities of vehicle C.G., respectively.
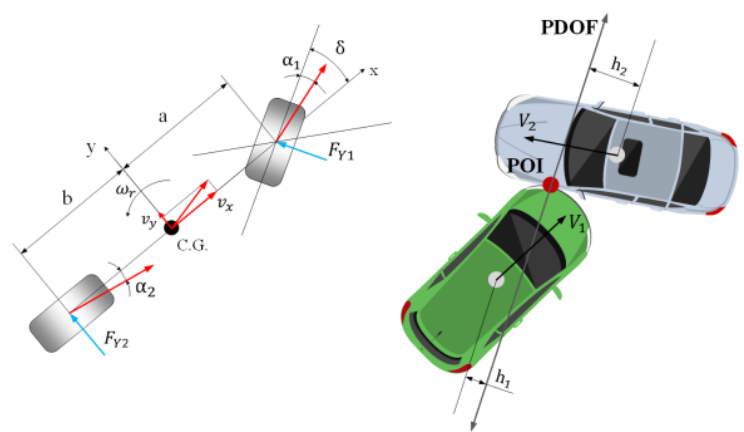

Fig. 4. 2 DoF Model (left) and Impact Force Diagram (right)

\section{Crash Severity Index Model for Optimal Path Selection}

Given the ego vehicle states along a specific trajectory, along with the predicted obstacle vehicle states, the unavoidable collision will be evaluated with the crash severity index (CSI) model proposed in [21], as shown in (4). The main idea is to obtain CSI according to the proportion of energy absorption in a collision, which is determined by the physical properties, motion states, and the geometric relationship of both vehicles in the predicted collision, as shown in Fig. 4.

$$
C S I=\frac{E E S_{1}}{V_{C P D O F}}=\sqrt{\frac{\gamma_{1} \gamma_{2}\left(1-\epsilon_{i}^{2}\right)}{\left(1+R_{K}\right)\left(\gamma_{2}+\gamma_{1} R_{m}\right)^{\prime}}}
$$

where $E E S_{1}$ is the energy equivalent speed of vehicle 1 (ego vehicle), $V_{C P D O F}$ is the velocity in the principal direction of force (PDOF) of both vehicles, $\epsilon_{i}$ is the coefficient of restitution, $R_{k}=K_{1} / K_{2}$, is the stiffness ratio of the two vehicles, $R_{m}=\mathrm{m}_{1} / \mathrm{m}_{2}$ is the mass ratio of the two vehicles. For each vehicle, the factor of mass reduction $\gamma$ is defined as $\gamma=\rho^{2} /\left(\rho^{2}+h^{2}\right)$, where $\rho$ is the radius of gyration of vehicle and $h$ is the force arm as depicted in Fig. 4.

For detailed calculation of each predicted crash corresponding to a path sample, the vehicle-to-vehicle contact point at the beginning, namely point of impact (POI), is taken as the collision point for subsequent force analysis [22-24]. After determining the contact point, we need to determine the PDOF, then the force arms and other parameters of the two vehicles can also be obtained. As shown in (4), the crash momentum index, defined as $C M I=\gamma_{1} \gamma_{2}\left(1+\epsilon_{i}\right) /\left(\gamma_{2}+\gamma_{1} R_{m}\right)$, has a great influence on the CSI, while the key variable to determine the magnitude of $\gamma$ is the PDOF in collision. To this end, the PDOF can be solved by the velocity change of the vehicle according to the momentum conservation law, i.e.

$$
\left\{\begin{array}{c}
R_{m} v_{10 x}+v_{20 x}=R_{m} v_{1 x}+v_{2 x} \\
R_{m} v_{10 y}+v_{20 y}=R_{m} v_{1 y}+v_{2 y} \\
\epsilon_{i, P D O F \|}=\frac{v_{2 \|}-v_{1 \|}}{v_{10 \|}-v_{20 \|}}=\epsilon_{i} \\
\epsilon_{i, P D O F \perp}=\frac{v_{2 \perp}-v_{1 \perp}}{v_{10 \perp}-v_{20 \perp}} \approx 1 \\
P D O F=\tan ^{-1}\left(\frac{v_{1 y}-v_{10 y}}{v_{1 x}-v_{10 x}}\right)
\end{array},\right.
$$

The descriptions of the symbols in the equations related to vehicle 1 are listed in Table I. For vehicle 2, the definitions of $\mathrm{m}_{2}, v_{20 x}, v_{20 y}, v_{2 x}, v_{20 \|}, v_{2 \|}, v_{20 \perp}, v_{2 \perp}$ are similar to those of vehicle 1 .

TABLE I

SYMBOLS AND DESCRIPTIONS OF THE CSI MODEL

\begin{tabular}{cl}
\hline \hline Symbols & \multicolumn{1}{c}{ Descriptions } \\
\hline $\mathrm{m}_{1}$ & mass of the vehicle 1 \\
$\epsilon_{i, P D O F \|}$ & coefficient of restitution parallel to the PDOF \\
$\epsilon_{i, P D O F \perp}$ & coefficient of restitution perpendicular to the PDOF \\
$v_{10 x}$ & pre-crash velocity of vehicle 1 in the $x$ direction \\
$v_{10 y}$ & pre-crash velocity of vehicle 1 in the $y$ direction \\
$v_{1 x}$ & post-crash velocity of vehicle 1 in the $x$ direction \\
$v_{1 y}$ & post-crash velocity of vehicle 1 in the $y$ direction \\
$v_{10 \|}$ & pre-crash velocity of vehicle 1 in parallel to the PDOF \\
$v_{10 \perp}$ & pre-crash velocity of vehicle 1 in perpendicular to the PDOF \\
$v_{1 \|}$ & post-crash velocity of vehicle 1 in parallel to the PDOF \\
$v_{1 \perp}$ & post-crash velocity of vehicle 1 in perpendicular to the \\
& PDOF
\end{tabular}

Usually an actual vehicle-to-vehicle collision lies between the completely-elastic collision and the completely-plastic collision, so the momentum changes in the PDOF and its perpendicular directions are not the same, i.e. $\epsilon_{i, P D O F \|} \neq$ $\epsilon_{i, P D O F \perp}$. The coefficient of restitution in the direction perpendicular to PDOF is approximately 1.0, i.e. $\epsilon_{i, P D O F \perp}=$ 1.0 , meaning a completely elastic collision [25].

To solve the five unknowns in the nonlinear collision equations (5), i.e. $v_{1 x}, v_{1 y}, v_{2 x}, v_{2 y}$, and PDOF, a numerical iterative method of computing is necessary, but it cannot meet 
the real-time requirements in applications. Therefore, the deep-learning based method is introduced to speed up the solving of collision equation.

\section{Neural Network Based Solution for Non-linear Equations}

The main idea is to construct and train a neural network to approximate the accurate but less-efficient numerical approach in solving (5) for the CSI index. To this end, Eq. (5) is firstly rewritten into the following form.

$$
\left\{\begin{array}{l}
R_{m} v_{10 x}+v_{20 x}=R_{m} v_{1 x}+v_{2 x} \\
R_{m} v_{10 y}+v_{20 y}=R_{m} v_{1 y}+v_{2 y} \\
\frac{\left(v_{2 x}+k_{0} v_{2 y}\right)-\left(v_{1 x}+k_{0} v_{1 y}\right)}{\left(v_{10 x}+k_{0} v_{10 y}\right)-\left(v_{20 x}+k_{0} v_{20 y}\right)}=\epsilon_{i} \\
\frac{\left(k_{0} v_{2 x}+v_{2 y}\right)-\left(k_{0} v_{1 x}+v_{1 y}\right)}{\left(k_{0} v_{10 x}+v_{10 y}\right)-\left(k_{0} v_{20}+v_{20}\right)}=1 \\
k_{0}\left(v_{1 x}-v_{10 x}\right)=v_{1 y}-v_{10 y}
\end{array},\right.
$$

where $k_{0}$ is the tangent value of PDOF. If we know the angle relationship between two vehicles, we can rewrite the equations even further using $v_{20 x}=v_{20} \cos (\theta)$ and $v_{20 y}=v_{20} \sin (\theta)$, where $\theta$ is the angle between the vehicle velocity and its heading direction ( $x$ direction). Then the solution of $k_{0}$ can work as the pivot for the final solution of (5).

Therefore, for the neural network, the inputs are $v_{10 x}, v_{10 y}$, $v_{20}, R_{m}, \theta$ and the output is $k_{0}$. In designing the network, the number of layers cannot be too few for approximation of the nonlinear equation (5). On the other hand, this number should be limited to prevent overfitting and the loss of the gradient. In this application example, the final network constructed has 6 hidden layers and the overall structure is shown in Fig. 5. The sigmoid function is used for the first two layers to meet the requirements of nonlinearization, while the ReLU function is used for the rest of layers to achieve more efficient gradient descent, and also to prevent overfitting or loss of gradient.

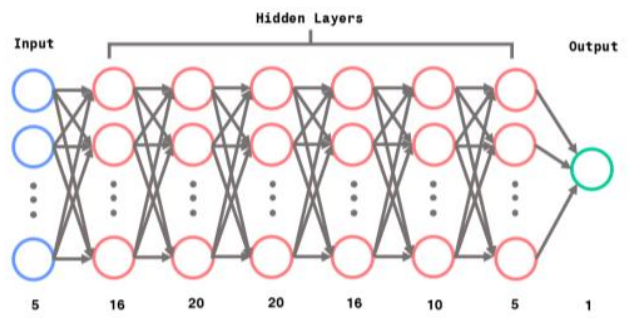

Fig. 5. Neural Network Structure Map

The training and evaluation datasets are constructed by using the sampled input conditions and ground-truth outputs, which can cover the operating conditions in application with different combinations of $v_{10 x}, v_{10 y}, v_{20}, R_{m}$ and $\theta$. To be specific, the obstacle vehicle speed $v_{20}$ is between $10 \mathrm{~m} / \mathrm{s}$ and $30 \mathrm{~m} / \mathrm{s}$, while $\theta$ is between 0 and $180^{\circ}$. The ground-truth $k_{0}=\tan (P D O F)$ is numerically solved from (5) via Matlab Optimization Toolbox.

For implementation, Tensorflow and Keras are used to construct and deploy the network. Since the distribution of output is not stable, the normalization operation is applied to the output data. For early stopping settings, the patience is 20 epochs and the minimum delta is $10^{-2}$. The loss function is defined as $M S E=\sum_{i=1}^{n}\left|y_{i}-y_{i}^{p}\right|^{2} / n$, where $n$ is the sample number, $y_{i}$ and $y_{i}^{p}$ are the ground-truth and predicted outputs, respectively. After training the neural network, the training loss and validation loss are shown in Fig.6, which can satisfy the prediction accuracy requirements in real applications.

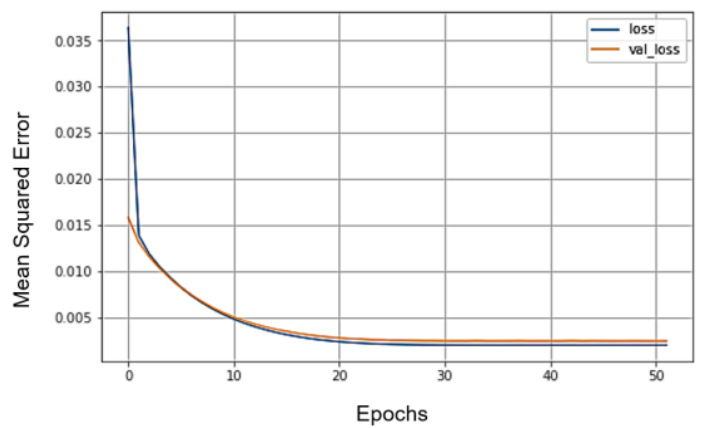

Fig. 6. Training and Evaluation Errors of Neural Network Prediction

Finally, the trained network is deployed in an industrial personal computer (IPC), which uses Intel Xeon Processor E3-1275, 32G RAM and GTX 1080 GPU. With the neural network, each prediction of CSI takes only $0.05 \mathrm{~s}$, meaning that the computing efficiency is ten times better than the numerical iteration method.

\section{E. Model Predictive Control (MPC) for Path Tracking}

After selecting the CSI-optimal path, the reference lateral position and yaw angle are to be tracked with MPC, while considering actuator constraints. The nonlinear vehicle kinematic model is given in (7). The system states are chosen as $\boldsymbol{x}=\left[\begin{array}{llll}X & Y & \theta & v\end{array}\right]^{T}$, the control inputs include demanded acceleration and steering angle $\boldsymbol{u}=[a \delta]^{T}$, where $X, Y$ indicate the vehicle's longitudinal and lateral position in the global coordinate system, respectively. $\theta$ is the yaw angle; $v$ is the longitudinal velocity, $a$ is the vehicle longitudinal acceleration.

$$
\left\{\begin{array} { c } 
{ \dot { X } = v \operatorname { c o s } \theta } \\
{ \dot { Y } = v \operatorname { s i n } \theta } \\
{ \dot { \theta } = v \operatorname { t a n } \delta / L } \\
{ \dot { v } = a }
\end{array} \Rightarrow \left\{\begin{array}{l}
\dot{\boldsymbol{x}}=\boldsymbol{f}(\boldsymbol{x}, \boldsymbol{u}) \\
\boldsymbol{y}=\boldsymbol{g}(\boldsymbol{x}, \boldsymbol{u})^{\prime}
\end{array}\right.\right.
$$

By linearizing at nominal conditions, we have

$$
\left\{\begin{array}{l}
\dot{\boldsymbol{x}}=\boldsymbol{A}_{c} \boldsymbol{x}+\boldsymbol{B}_{c} \boldsymbol{u} \\
\boldsymbol{y}=\boldsymbol{C}_{c} \boldsymbol{x}+\boldsymbol{D}_{c} \boldsymbol{u}
\end{array}\right.
$$

where $\boldsymbol{A}_{c}, \boldsymbol{B}_{c}, \boldsymbol{C}_{c}, \boldsymbol{D}_{c}$ are as follow.

$$
\begin{gathered}
\boldsymbol{A}_{c}=\left[\begin{array}{cccc}
0 & 0 & -v \sin \theta & \cos \theta \\
0 & 0 & v \cos \theta & \sin \theta \\
0 & 0 & 0 & \tan \delta / L \\
0 & 0 & 0 & 0
\end{array}\right], \boldsymbol{B}_{c}=\left[\begin{array}{cc}
0 & 0 \\
0 & 0 \\
0 & \left(1+\tan ^{2} \delta\right) v / L \\
1 & 0
\end{array}\right] \\
\boldsymbol{C}_{c}=\left[\begin{array}{cccc}
0 & 1 & 0 & 0 \\
0 & 0 & 1 & 0
\end{array}\right], \boldsymbol{D}_{c}=\mathbf{0}_{2 \times 2} .
\end{gathered}
$$

Then the discrete state-space model corresponding to (8) is

$$
\left\{\begin{array}{c}
\boldsymbol{x}(k+1)=\boldsymbol{A}_{d} \boldsymbol{x}(k)+\boldsymbol{B}_{d} \boldsymbol{u}(k) \\
\boldsymbol{y}(k)=\boldsymbol{C}_{d} \boldsymbol{x}(k)+\boldsymbol{D}_{d} \boldsymbol{u}(k)
\end{array}\right.
$$

where $\boldsymbol{A}_{d}=e^{\boldsymbol{A}_{c} T_{s}}, \boldsymbol{B}_{d}=\int_{k T_{s}}^{(k+1) T_{s}} e^{\boldsymbol{A}_{c}\left[(k+1) T_{s}-\tau\right]} \boldsymbol{B}_{c} d \tau, \boldsymbol{C}_{d}=$ $\boldsymbol{C}_{c}, \boldsymbol{D}_{d}=\boldsymbol{D}_{c}$, and $T_{s}$ is the sampling interval. 
For practical applications, actuator limits should be set according to steering, braking and traction capabilities and vehicle operating conditions. Considering the actuator limits of the experiment vehicle, a maximum front steering wheel angle is set as $25^{\circ}$, maximum steering rate as $50^{\circ} / \mathrm{s}$, maximum braking deceleration as $-0.7 \mathrm{~g}$, and maximum acceleration as $0.5 \mathrm{~g}$.

$$
\left\{\begin{aligned}
-25^{\circ} & \leq \delta \leq 25^{\circ} \\
-50^{\circ} / \mathrm{s} & \leq \dot{\delta} \leq 50^{\circ} / \mathrm{s} \Rightarrow \boldsymbol{u}_{\min } \leq \boldsymbol{u} \leq \boldsymbol{u}_{\max } \\
-0.7 \mathrm{~g} & \leq a \leq 0.5 \mathrm{~g}
\end{aligned}\right.
$$

The desired system outputs $\boldsymbol{y}_{\text {des }}$ are the lateral position $y_{d e s}$ and the yaw angle $\theta_{d e s}$, i.e. $\boldsymbol{y}_{\text {des }}=\left[y_{d e s} \theta_{d e s}\right]^{\mathrm{T}}$. Then the final cost is expressed as the squared error between the desired and the observed outputs. Finally, the tracking problem of the CSI-optimal path is formulated and solved as a linear MPC problem by combining (9) to (11).

$$
\begin{gathered}
\min J=\min _{\boldsymbol{u}}|| \boldsymbol{y}_{\text {des }}-\boldsymbol{y} \|\left.\right|^{2}, \\
\text { s.t. } \boldsymbol{u}_{\min } \leq \boldsymbol{u} \leq \boldsymbol{u}_{\max }
\end{gathered}
$$

\section{VALIDATION VIA SIMULATION AND EXPERIMENT}

For case studies, two emergency scenarios are selected, i.e. intersection crossing and entry ramp merging, which are typical in urban driving. Another reason is that the potential impact patterns in these two scenarios include oblique collisions that can be well handled with the adopted CSI model, but are too complex for those popular Delta-V crash severity models.

On the other hand, real vehicle experiments, especially in high speed crash imminent scenarios are dangerous and need professional facilities. Therefore, simulations are carried out for both cases, but only the intersection case is further validated through experiment. Key parameters used in the validation process are shown in Table II.

TABLE II

PARAMETERS OF VEHICLES

\begin{tabular}{clllll}
\hline \hline Parameter & Value & Unit & Parameter & Value & Unit \\
\hline \hline $\mathrm{m}_{1}$ & 1575 & $\mathrm{~kg}$ & $\rho_{1}, \rho_{2}$ & 1.38 & $\mathrm{~m}$ \\
$\mathrm{~m}_{2}$ & 1500 & $\mathrm{~kg}$ & Length & 4 & $\mathrm{~m}$ \\
$R_{k}$ & 1 & & Width & 2 & $\mathrm{~m}$ \\
$\epsilon_{i}$ & 0.9 & - & $\mathrm{a}_{1}, \mathrm{~b}_{1}$ & $1.2,1.6$ & $\mathrm{~m}$
\end{tabular}

\section{A. Entry Ramp Merging Scenario}

The entry ramp merging scenario shown in Fig. 1 is firstly simulated and analyzed. When the ego vehicle is driving straight forward (at $10 \mathrm{~m} / \mathrm{s}$ ) and passing a ramp entry, the speeding vehicle on the ramp merges to the main lane aggressively (at $20 \mathrm{~m} / \mathrm{s}$ ), which leads to an unavoidable collision. The trajectory sampling and crash severity prediction results are shown in Fig. 7.

According to the CSI results, if no active braking or evasive steering is activated, CSI is about 0.19, i.e. the zero-steering result in the green CSI curve. Interestingly, a full-braking-only strategy (i.e. $a_{x}=-0.7 \mathrm{~g}$ ) does not reduce but rather increase the crash severity index, with the maximum CSI $>0.2$ (the dashed orange line). This result is even worse than doing nothing, meaning that if not considering the crash severity model in the normal emergency braking assistant system, the ultimate goal of crash avoidance and mitigation is not achievable at all.
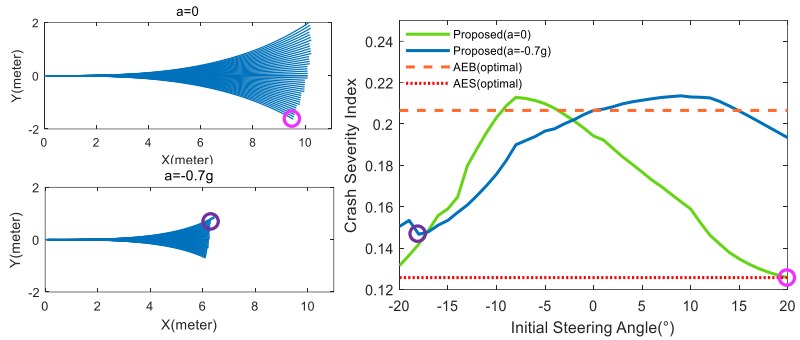

Fig. 7. Results of Different Strategies in Entry Ramp Merging Scenario

As shown in Fig. 7, both "braking and steering to left" (blue curve) and "only steering to right" (green curve) can reduce the crash severity, though both strategies actually lead to different patterns of collision impact. For the braking and steering to left strategy, the minimum CSI is 0.142 with $a_{x}=-0.7 \mathrm{~g}$ and the initial steering angle $\delta_{0}$ about -18 degrees. If the vehicle adopts the steering-only strategy, the lowest crash severity $\mathrm{CSI}=0.125$ can be achieved by 20 degrees initial steering to the right, which ends with a right-front side impact with the obstacle vehicle. However, this steering-only strategy will pull the vehicle out of the lane, which certainly is risky if considering the traffic law. Therefore, the decision of braking and steering to left is better to minimize the risk, i.e. by still staying in the lane and lowering the CSI.

Such dilemmas of strategy decision are not rare in emergency scenarios, which again proves that an optimal crash mitigation strategy is closely related to the dynamic states and poses of both vehicles. It is understandable that different planning strategies may lead to different forms of collisions, but the fast evaluation of CSI can work as a criterion for fair comparisons among the sampled paths. This is extremely useful in avoiding a too risky (or even wrong) decision of path.

\section{B. Intersection Crossing Scenario}

The intersection crossing scenario is shown in Fig. 8, where the ego vehicle (in green) approaches the intersection with a speed of $10 \mathrm{~m} / \mathrm{s}$, and its initial distance from the intersection center is $7 \mathrm{~m}$. Due to the occlusion by a stopped truck, the aggressive vehicle (in silver) cannot be detected before intruding into the intersection at an extremely high speed of $30 \mathrm{~m} / \mathrm{s}$. The TTC analysis shows that the high speed of obstacle vehicle will lead to an unavoidable collision. For this scenario, the simulation and digital-twin experiment are carried out.

\section{1) Simulation}

Based on the proposed CSI-optimal approach, Fig. 9 presents the clothoid trajectory samples for steering-only $\left(a_{x}=0\right)$ and braking-steering $\left(a_{x}=-0.7 \mathrm{~g}\right)$ strategies. If the vehicle does not decelerate at all, i.e. it adopts only AES steering evasion (the green curve), an optimal selection of trajectory is steering to the left, which leads to an oblique rear-end collision with a minimum CSI as 0.16. If only AEB is activated, there will still be a side-impact collision, but the estimated CSI is lowered to 0.15 with maximum $-0.7 \mathrm{~g}$ braking. This indicates AES-only strategy may work well to avoid a collision in some scenarios, 
but it is still risky in mitigating the crash severity for an unavoidable collision.

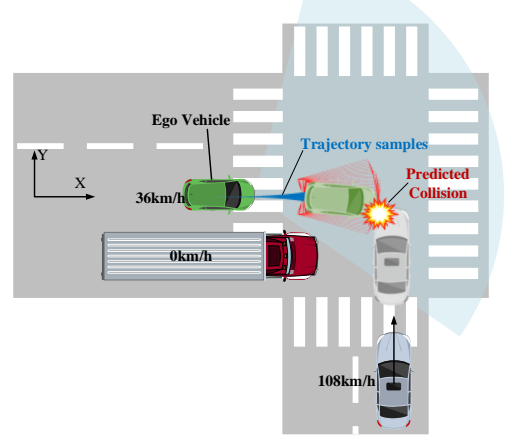

Fig. 8. Emergency Scenario at Intersection
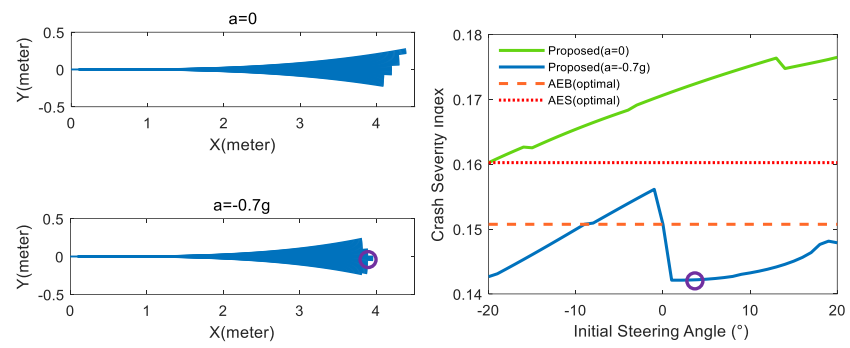

Fig. 9. Results of Different Strategies in Intersection Scenario

If using combined braking and steering, e.g. $a_{x}=-0.7 \mathrm{~g}$, the crash severity index can be further lowered to 0.142 , meaning that integrated AES and AEB functions can greatly reduce the collision injury. In such dynamic emergency scenarios, the rugged bends in the CSI estimation results of the integrated AES and AEB strategy is due to the change of impact pattern, i.e. at which positions and angles that both vehicles collide. Note that it is also found that a rapid acceleration (e.g. $\left.a_{x}>0.3 \mathrm{~g}\right)$ is even possible to avoid a collision $(\mathrm{CSI}=0)$, but due to the high risk it is not recommended to take such aggressive measures. Therefore, a reliable choice of optimal path corresponds to 2-5 degrees of steering to right, as indicated in Fig. 8 (the circle).

\section{2) Experiment}

The digital-twin experiment is schemed in Fig. 10. Based on MSC CarSim software running in the industrial personal computer, the ego vehicle is simultaneously presented in the virtual and physical worlds, while the obstacle vehicle and the unavoidable collisions only happen in the virtual world. The states of both vehicles are fed in real time to IPC, where the proposed motion planning and control algorithms are deployed with Matlab/Simulink. For the tracking control, a high-definition GNSS/IMU, i.e. CGI-610 by CHCNAV, is used to feed back the vehicle lateral position and yaw angle to the IPC. Then the lower-level controller in NI-CRIO is to realize the closed loop regulation of acceleration and steering, which is further realized in the experiment vehicle. A section of two-lane-two-way road, totally $200 \mathrm{~m}$, is selected as the experiment site.

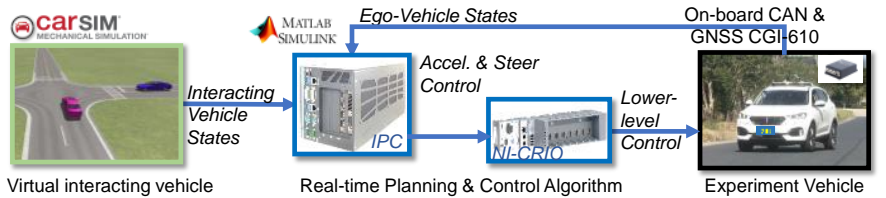

Fig. 10. Digital-twin Experiment of Intersection Case

During the interaction process, the CSI-optimal planning strategy from the above simulation is realized in real time in the vehicle. The results of the intersection crossing experiment are shown in Fig. 11, which includes the vehicle lateral positions, yaw angles, steering wheel angle and longitudinal accelerations. The two vehicles collide at $0.47 \mathrm{~s}$, which means that it is almost impossible to fully avoid the collision by any strategies, either planning or control. This is confirmed by the acceleration and steering control inputs, which are both constrained due to actuator limits. The steering handwheel angle only climbs after 0.16 s of pure delay, while the braking deceleration achieves about $0.15 \mathrm{~g}$ before the collision happens, although the braking pressure building is not fully finished.
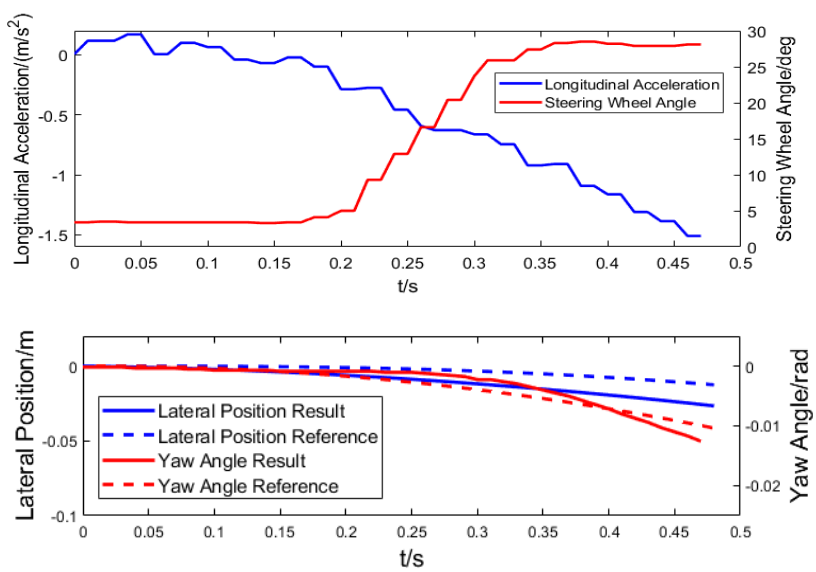

Fig. 11. Real Vehicle Experiment Results of Intersection Scenario

According to Fig. 9, the decision of braking and steering to left will increase CSI, which should not be adopted. In the real-time vehicle experiment, the braking and slight steering to right is achieved as shown in Fig. 11. The errors of both the lateral deviation and the yaw angle are acceptably small. Note that due to the limits of our testing ground facility, higher speed experiments, which need larger safe space, are not possible in this work. However, the real vehicle deployment can show that the safety and real-time performances of the proposed motion planning and control algorithm can be guaranteed.

\section{Sensitivity Analysis}

To check how the uncertainties of parameters and vehicle states in the CSI modelling affect the final planning strategies, the sensitivity analysis of mass ratio $R_{m}$, coefficient of restitution $\epsilon_{i}$ and obstacle vehicle speed $v_{20}$ on the crash severity index is further carried out. Note that although the state-of-the-art technologies of obstacle vehicle motion prediction has achieved a high level, it is still necessary to consider abrupt driving behaviors of the interacting vehicle, e.g. strong braking or even accelerating when approaching the entry ramp. 
Here the intersection scenario is taken as an example, i.e. $v_{10}=10 \mathrm{~m} / \mathrm{s}$ and $a_{x}=-0.7 \mathrm{~g}$. As shown in Fig. 12, the different estimates of $R_{m}$ or $\epsilon_{i}$ will affect the CSI magnitude, but the best path selection, i.e. the shaded area, is not affected.
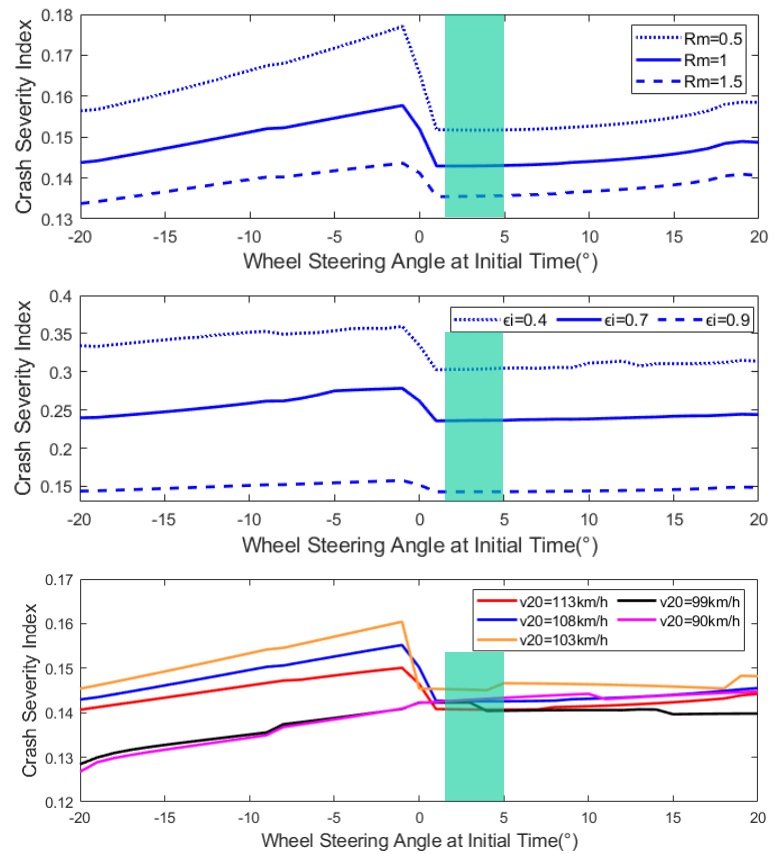

Fig. 12. Sensitivity Analysis of Mass, Stiffness and Obstacle Speed

The shape of the curves may change as the vehicle speed changes. When the vehicle speed does not change much $\left(v_{20} \pm\right.$ $5 \mathrm{~km} / \mathrm{h}$ ), the final path decision does not change much. However, when the obstacle vehicle decelerates to $99 \mathrm{~km} / \mathrm{h}$ (i.e. with $-0.5 \mathrm{~g}$ deceleration) or $90 \mathrm{~km} / \mathrm{h}$ (i.e. with $-1 \mathrm{~g}$ deceleration), the final path decision should be steering to left as much as possible, if the steering actuator allows. This is because the rapid reduction of $v_{20}$ will change the collision POI, i.e. the ego vehicle will be side-impacted by the obstacle vehicle. Even under these two extreme conditions of obstacle speed, the selected path with slight steering to right, shaded in Fig.10, is still acceptable with a low CSI. This shows that in the application example, the CSI-optimal planning approach can perform reliably safe even with model uncertainties. Note that the aim of proposed planning approach is to try best in minimizing fatalities and injuries using the crash severity modelling. It is understandable that for real applications, such uncertainties of both vehicles should be carefully simulated and experimentally validated.

\section{CONCLUSION}

In this paper, we propose a motion planning algorithm to handle the scenarios where a collision cannot be avoided completely. The clothoid curve is used to do the path sampling, from which the generalized crash severity index of the collision is introduced to find an optimal or sub-optimal trajectory. Simulation and real vehicle experiment of emergency scenarios, show that the proposed algorithm can provide a promising solution in crash mitigation in unavoidable collisions.

This work is a step further towards safe motion planning for automated driving, especially in emergency scenarios. Here we mainly focus on introducing a more generalized crash severity model to the motion planning algorithm development.
However, it is still in the early concept stage with only limited validation of emergency scenarios. Although the adopted crash severity model has been validated with real accident data, for practical applications the real crashworthiness properties and test data can be used to calibrate the CSI model of one specific vehicle. Due to the data availability issues, this is yet not possible for us but may be considered in the future work.

\section{REFERENCES}

[1] D. González, J. Pérez, V. Milanés and F. Nashashibi, "A Review of Motion Planning Techniques for Automated Vehicles," in IEEE Transactions on Intelligent Transportation Systems, vol. 17, no. 4, pp. 1135-1145, April 2016, doi: 10.1109/TITS.2015.2498841.

[2] W. Schwarting, J. Alonso-Mora, and D. Rus, "Planning and decision-making for autonomous vehicles," Annual Review of Control, Robotics, and Autonomous Systems, vol. 1, pp. 187-210, 2018.

[3] L. Claussmann, M. Revilloud, D. Gruyer, and S. Glaser, "A review of motion planning for highway autonomous driving," IEEE Transactions on Intelligent Transportation Systems, vol. 21, no. 5, pp. 1826-1848, 2019.

[4] K. Lee and D. Kum, "Collision Avoidance/Mitigation System: Motion Planning of Autonomous Vehicle via Predictive Occupancy Map," in IEEE Access, vol. 7, pp. 52846-52857, 2019, doi: 10.1109/ACCESS.2019.2912067.

[5] D. Shen, Y. Chen, L. Li, and S. Chien, "Collision-Free Path Planning for Automated Vehicles Risk Assessment via Predictive Occupancy Map," in 2020 IEEE Intelligent Vehicles Symposium (IV), 2020, pp. 985-991.

[6] S. Feng, Y. Qian, and Y. Wang. "Collision Avoidance Method of Autonomous Vehicle Based on Improved Artificial Potential Field Algorithm." Proceedings of the Institution of Mechanical Engineers, Part D: Journal of Automobile Engineering 235, no. 14, December 2021: 3416-30.

[7] H. Wang, Y. Huang, A. Khajepour, Y. Zhang, Y. Rasekhipour and D. Cao, "Crash Mitigation in Motion Planning for Autonomous Vehicles," in IEEE Transactions on Intelligent Transportation Systems, vol. 20, no. 9, pp. 3313-3323, Sept. 2019, doi: 10.1109/TITS.2018.2873921.

[8] V. Khattar and A. Eskandarian. "Reactive Online Motion Re-Planning for Crash Mitigation in Autonomous Vehicles Using Bezier Curve Optimization." Proceedings of the ASME 2020 International Mechanical Engineering Congress and Exposition, vol. 7B: Dynamics, Vibration, and Control, November 16-19, 2020.

[9] Y. Qin, E. Hashemi and A. Khajepour, "Integrated Crash Avoidance and Mitigation Algorithm for Autonomous Vehicles," IEEE Transactions on Industrial Informatics, doi: 10.1109/TII.2021.3058948.

[10] B. Simon, F. Franke, P. Riegl, and A. Gaull, "Motion planning for collision mitigation via fem-based crash severity maps," in 2019 IEEE Intelligent Vehicles Symposium (IV), 2019, pp. 2187-2194.

[11] M. Parseh, F. Asplund, L. Svensson, W. Sinz, E. Tomasch, and M. Torngren, "A Data-Driven Method Towards Minimizing Collision Severity for Highly Automated Vehicles," IEEE Transactions on Intelligent Vehicles, 2021.

[12] M. Huang, Vehicle crash mechanics. CRC press, 2002

[13] S. Kambhampati and L. Davis, "Multiresolution path planning for mobile robots," in IEEE Journal on Robotics and Automation, vol. 2, no. 3, pp. 135-145, September 1986, doi: 10.1109/JRA.1986.1087051.

[14] J. J. Kuffner and S. M. LaValle, "RRT-connect: An efficient approach to single-query path planning," Proceedings of 2000 IEEE Int. Conf. on Robotics and Automation, San Francisco, CA, USA, 2000, doi: 10.1109/ROBOT.2000.844730.

[15] Y. Guan, K. Yokoi, O. Stasse and A. Kheddar, "On robotic trajectory planning using polynomial interpolations," 2005 IEEE International Conference on Robotics and Biomimetics - ROBIO, Hong Kong, China, 2005, pp. 111-116, doi: 10.1109/ROBIO.2005.246411.

[16] H. Hoang, A. Khoa Tran, L. Nhat Thai Tran, M. -H. Le and D. -T. Tran, "A Shortest Smooth-path Motion Planning for a Mobile Robot with Nonholonomic Constraints," 2021 International Conference on System Science and Engineering (ICSSE), 2021, pp. 145-150, doi: 10.1109/ICSSE52999.2021.9538414.

[17] J. A. Reeds and L. A. Shepp. "Optimal paths for a car that goes both forwards and backwards." Pacific Journal of Mathematics, 145, 1990: 367-393. 
[18] F. A. Berg, F. Walz, M. Muser, H. Buerkle and J. Epple, "Implications of velocity change Delta-V and energy equivalent speed EES for injury mechanism assessment in various collision configurations," Proceedings of the 1998 Int. IRCOBI Conf. on the Biomechanics of Impact, Gothenburg, Sweden, 1998, pp. 57-72.

[19] A. Ji, and D. Levinson, "An Energy Loss-Based Vehicular Injury Severity Model." Accident Analysis \& Prevention, vol. 146, October 2020, p. 105730. doi:10.1016/j.aap.2020.105730.

[20] M. Paul and I. Ghosh. "Development of conflict severity index for safety evaluation of severe crash types at unsignalized intersections under mixed traffic", Safety Science, vol. 144, December 2021, doi: 10.1016/j.ssci.2021.105432.

[21] D. Vangi, "Impact Severity Assessment in Vehicle Accidents." International Journal of Crashworthiness, vol. 19, no. 6, 2014, pp. 57687. doi:10.1080/13588265.2014.921974.

[22] M. Gulino, L. Gangi, A. Sortino and D. Vangi, "Injury Risk Assessment Based On Pre-Crash Variables: The Role Of Closing Velocity And Impact Eccentricity". Accident Analysis \& Prevention, vol 150, 2021, p. 105864. Elsevier BV, doi:10.1016/j.aap.2020.105864.

[23] D. Vangi, M. Gulino, A. Fiorentino and A. Virga, "Crash Momentum Index and Closing Velocity as Crash Severity Index." Proceedings of the Institution of Mechanical Engineers, Part D: Journal of Automobile Engineering, vol. 233, no. 13, 2019, pp. 3318-26. doi:10.1177/0954407018823658.

[24] K. Kusano and H. Gabler, "Automated Crash Notification: Evaluation of in-Vehicle Principal Direction of Force Estimations." Transportation Research Part C: Emerging Technologies, vol. 32, 2013, pp. 116-28. doi:10.1016/j.trc.2012.09.005.

[25] M. Savova-Mratsenkova and G. Djonev. "Determining the Values of the Coefficient of Restitution in the Meanwhile of a Crash between Two Vehicles." IOP Conf. Series: Materials Science and Engineering, vol. 618, 2019, p. 012058. doi:10.1088/1757-899x/618/1/012058.

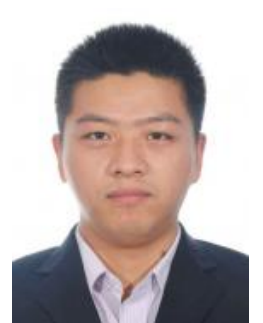

Daofei $\mathbf{L i}$ received the B.S. degree in Vehicle Engineering from the Jilin University, Changchun, China, in 2003, and the Ph.D. degree in Vehicle Engineering from the Shanghai Jiao Tong University, Shanghai, China, in 2008.

In 2008, he joined the Institute for Power Machinery and Vehicular Engineering, Faculty of Engineering, Zhejiang University (PMVEI, ZJU). In 2011, he was a Visiting Scholar with the University of Missouri-Columbia. From 2014 to 2016, he was a Visiting Scholar with the University of Michigan, Ann Arbor, Michigan. Since 2016, he has been Associate Professor with PMVEI, ZJU. He currently directs the Research Group of Human-Mobility-Automation, and his research interests include vehicle dynamics and control, intelligent vehicle and complete human-vehicle system dynamics.

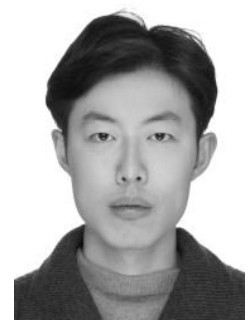

Zhaohan Hu is currently an undergraduate student of Zhejiang University. $\mathrm{He}$ is expected to receive his Bachelor's degree of vehicle engineering in 2022.

$\mathrm{He}$ is with the Research Group of Human-Mobility-Automation, PMVEI, ZJU. His research interests include motion planning and driver behavior modelling. 